\title{
Actividad antiproliferativa in vitro, de los complejos de paladio(II) incluidos en la $\beta$-ciclodextrina, frente a varias líneas de células tumorales de humano
}

\author{
Nancy Jaimes ${ }^{1}$, Siham Salmen ${ }^{2}$, Melisa Carolina Colmenares ${ }^{3}$ y Rosa Virginia Mendoza ${ }^{3}$ \\ ${ }^{1}$ Grupo de Investigación en Biología Molecular y Genética, Universidad de Pamplona, Colombia, \\ ${ }^{2}$ Instituto de Inmunología Clínica, Facultad de Medicina, Universidad de Los Andes, Mérida, \\ Venezuela. \\ ${ }^{3}$ Centro de Microscopía Electrónica “Dr Ernesto Palacios Prü”. Universidad de Los Andes, Mérida, \\ Venezuela.
}

Palabras clave: complejos de paladio(II); $\beta$-ciclodextrina; HeLa; HCT-15; PC-3.

Resumen. Las propiedades antineoplásicas de las tiosemicarbazonas y sus complejos de paladio(II), se han convertido en un importante tema de investigación, debido a que las actividades citotóxicas son prometedoras en la oncología. El objetivo de este trabajo fue evaluar el efecto antiproliferativo de los ligandos derivados del pirazol-3-carbaldehído(HL $\left.{ }^{1-2}\right)$, los complejos bis-quelatos de paladio(II) $\mathrm{Pd}\left(\mathrm{L}^{1}\right)_{2}$ y los complejos $\mathrm{Pd}\left(\mathrm{L}^{2}\right)_{2}$ incluidos en la ß-cliclodextrina (ß-CD), frente a líneas de células tumorales de humano, como HCT-15, PC-3 y HeLa; para lo cual se empleó el método de la sulforodamina B. Los resultados mostraron que la inclusión de los complejos $\mathrm{Pd}\left(\mathrm{L}^{1-2}\right)_{2}$ en la $\beta-\mathrm{CD}$, provocaba una disminución de los valores de $\mathrm{CI}_{50}$ frente a las diferentes líneas tumorales ensayadas. Los rangos encontrados de los valores de $\mathrm{CI}_{50}$ fueron desde 0,45 hasta $1,13 \mu \mathrm{M}$ para el complejo Pd(MePhPzTSC) 2 y desde 0,86 hasta $1,31 \mu \mathrm{M}$ para el complejo $\mathrm{Pd}\left(\mathrm{Ph}_{2} \mathrm{PzTSC}_{2}\right.$, mientras que cuando estos complejos bis-quelatos de paladio(II) se incluyeron en la $\beta-C D$, se evidenció un incremento de la actividad antiproliferativa $\left(\mathrm{CI}_{50}=0,14-0,52 \mu \mathrm{M}\right)$. Además, que se requirió 5 a 10 veces menos concentración de los complejos de paladio(II) solos, que cuando estos complejos estaban incluidos en la ß-CD. Los hallazgos también, indican que la línea celular HeLa, es más sensible para el compuesto $\mathrm{Pd}(\mathrm{MePhPzTSC}) \cdot \beta-\mathrm{CD}$, mientras que la línea celular HCT-15 fue sensible solamente para el compuesto $\mathrm{Pd}\left(\mathrm{Ph}_{2} \mathrm{PzTSC}\right)_{2} \bullet \beta-\mathrm{CD}$. En conclusión, de acuerdo a los hallazgos, la inclusión de los complejos de paladio(II) en la $\beta-C D$ aumenta su actividad antiproliferativa.

Autor de Correspondencia: Nancy Jaimes: Departamento de Biología, Ciudadela Universitaria - Edificio Jorge Gaitán, Pamplona, Norte de Santander, Colombia. Teléfono: 5685303-5685304 ext 243 Correo electronico: najame3@hotmail.com 


\title{
In vitro antiproliferative activity of palladium(II) complexes included within the $\beta$-cyclodextrin against various human tumor cell lines
}

Invest Clin 2018; 59(1): 17 - 27

Keywords: palladium(II); complexes; $ß$-ciclodextrine; HeLa; HCT-15; PC-3

\begin{abstract}
The antineoplastic properties of the thiosemicarbazones ligands and their palladium(II) complexes, have become an important research subject, due to the cytotoxic activities with very promising results in the oncology field. The aim of this work was to evaluate the antiproliferative effect of the ligands derived from pyrazole-3-carbaldehyde $\left(\mathrm{HL}^{1-2}\right)$, the palladium(II) bis-chelate $\left[\mathrm{Pd}\left(\mathrm{L}^{1}\right)_{2}\right]$ complexes and the $\left[\mathrm{Pd}\left(\mathrm{L}^{2}\right)_{2}\right]$ complexes included witin the B-cyclododextrin (ß-CD), against several human tumor cell lines such as HCT-15, PC-3 and HeLa, using the sulforhodamine B method. Our results showed that the inclusion of $\left[\mathrm{Pd}\left(\mathrm{L}^{1-2}\right)_{2}\right]$ complexes witin the $\beta$-CD caused a decrease in $\mathrm{IC}_{50}$ values against the different tumor lines tested. The ranges found of $\mathrm{IC}_{50}$ values were from 0.45 to $1.13 \mu \mathrm{M}$ for the $\left[\mathrm{Pd}(\mathrm{MePhPzTSC})_{2}\right]$ complex, and from 0.86 to $1.31 \mu \mathrm{M}$ for the $\left[\mathrm{Pd}\left(\mathrm{Ph}_{2} \mathrm{PzTSC}\right)_{2}\right]$ complex, whereas when these palladium(II) bis-chelate complexes were included in the $\beta-\mathrm{CD}$, an increase in antiproliferative activity $\left(\mathrm{IC}_{50}=\right.$ $0.14-0.52 \mu$ ) was evidenced. These results demonstrate that when the palladium(II) complexes are alone is required 5 to 10 times less concentration than when these complexes were included in the $\beta$-CD. Also, the obtained results in this study indicate that the HeLa cell line are more sensitive to the $\left[\mathrm{Pd}(\mathrm{MePhPzTSC})_{2}\right] \cdot \beta-\mathrm{CD}$ compound, whereas the HCT-15 cell line was only sensitive for the $\left[\mathrm{Pd}\left(\mathrm{Ph}_{2} \mathrm{PzTSC}\right)_{2}\right] \cdot \beta-\mathrm{CD}$ compound. In general, this study has shown that the inclusion of palladium(II) complexes on $\beta-\mathrm{CD}$ enhances its antiproliferative activity.
\end{abstract}

Recibido: 08-03-2017 Aceptado: 08-03-2018

\section{INTRODUCCION}

El cáncer en muchos casos puede resultar en la muerte del paciente debido a la resistencia o fallas de la quimioterapia. Por lo tanto, se requiere de nuevos enfoques terapéuticos más eficaces y con menos efectos tóxicos para quienes lo reciben. Los estudios in vitro desarrollados utilizando diferentes tipos de complejos de paladio han producido resultados prometedores $(1,2)$, y contribuyen al desarrollo de nuevos medicamentos contra el cáncer. Desde hace más de 30 años se conoce que los complejos de paladio tienen actividad anti-fúngica, anti-viral, 
anti-neoplásica y anti-bacteriana (3), pero el estudio de sus propiedades contra el cáncer se ha convertido en un tema de creciente interés en los últimos 15 años. Se han realizado estudios en diferentes tipos de líneas celulares de tumores sólidos y neoplasias malignas hematológicas, con resultados muy prometedores en el campo de la oncología (4). Se ha informado que la actividad antitumoral in vitro frente a células de mamífero que ejercen los agentes quelantes derivados de las tiosemicarbazonas heterocíclicas $(\mathrm{N}, \mathrm{N}, \mathrm{S})$, es debida a la inhibición de la biosíntesis del ADN, mediante el bloqueo de la enzima ribonucleótidoreductasa, esencial en la conversión de ribonucleótidos en desoxirribonucleótidos $(4,6)$ o en la inhibición de la tiorredoxinareductasa involucrada en la biosíntesis de nucleótidos; otro de los mecanismos propuestos informados, es que estas tiosemicarbazonas heterocíclicas actúan como agentes que se intercalan en las bases pirimidinas y purinas del $\mathrm{ADN}$, induciendo cambios en la conformación de la doble hélice, lo que conduce a la muerte celular y al bloqueo de la división de las células cancerosas (5, 7-10).

Los complejos de paladio(II) muestran mayor solubilidad que el cisplatino, ejemplo de ello, son los complejos de paladio(II) con ligandos oxima de ácido glioxílico, que muestran una mayor solubilidad que los complejos de platino(II) con el mismo ligando (11), lo que hace atractiva la síntesis de estos complejos en el futuro para su aplicación en las neoplasias. Las tiosemicarbazonas se han utilizado como ligandos quelatos debido a sus átomos donadores Ny S para la síntesis de complejosde paladio(II), $y$ se ha observado que estos complejos incrementan su actividad antitumoral (12). La preparación de los complejos con otros metales de transición con nuevos ligandos derivados de las tiosemicarbazonas, ha ocasionado una significativa atención debido a sus propiedades farmacológicas (13). Sin embargo, a pesar de que los complejos de paladio(II) con ligandos tiosemicarbazonas muestran una mayor actividad citotóxica in vitro, las dosis in vitro empleadas para lograr el efecto inhibitorio deseado en las células tumorales es muy variable y depende de la línea celular utilizada (14-18). Se ha evidenciado que los complejos de paladio(II) con ligandos tiosemicarbazonas, muestran mayor actividad citotóxica frente a las líneas celulares humanas: adenocarcinoma de mama (MCF-7) y adenocarcinoma de próstata $(\mathrm{PC}-3)\left(\mathrm{CI}_{50}=0,08 \mu \mathrm{M}\right)$, y frente a la línea celular humana de adenocarcinoma de cérvix uterino(HeLa), $\left(\mathrm{CI}_{50}>1,0 \mu \mathrm{M}\right)(15,17,19,20)$. Los complejos de paladio(II) con ligandos diferentes a las tiosemicarbazonas y con otros metales como el cobre o platino evidencian su efecto antiproliferativo con valor $\mathrm{CI}_{50}$ de 0,31 $\mu \mathrm{M}$ para la línea celular HCT-15, mientras que los valores $\mathrm{CI}_{50}$ para las líneas celulares de cáncer de colon HT-29 y HCT-116 se encuentran en el rango de $0,2 \mu \mathrm{M}-100 \mu \mathrm{M}$ y $0,003-10 \mu \mathrm{M}$, respectivamente. Estos resultados indican el gran potencial farmacológico que presentan estos complejos y que están relacionados con el tipo de tumor (1,2, 21-24). Además, estos complejos metálicos muestran diferencias significativas en los rangos de las concentraciones inhibitorias, que pueden estar asociadas a las divergencias estructurales entre los complejos, a la sensibilidad de las líneas celulares (18) o a la solubilidad tanto de los ligandos tiosemicarbazonas como de los complejos de paladio(II).

En los últimos años, la industria farmacéutica ha invertido más dinero en el desarrollo de compuestos que sirven como medios de transporte para los fármacos anticancerígenos, para así alcanzar el mismo efecto a más bajas dosis y con una menor toxicidad. Las ciclodextrinas $(\alpha$, $\beta, \gamma)$ son macromoléculas físicamente estables, producidas por la degradación enzimática del almidón, que tienen una superficie externa hidrofílica y un núcleo hidrofóbico, y en el

Vol. 59(1): 17 - 27, 2018 
caso de la $\beta-C D$, es el compuesto ideal por su tamaño, bajo costo y mayor disponibilidad (25). De hecho, su uso como medio de transporte para las drogas antineoplásicas, ha mostrado baja toxicidad (26), y un incremento en la muerte celular inducida por compuestos con actividad antineoplásica $(27,28)$.

Hoy en día, la quimioterapia sigue siendo la piedra angular en la oncología médica, por lo que la búsqueda de nuevos compuestos más eficaces y menos tóxicos es necesaria para mejorar la calidad de vida de los pacientes que padecen cáncer.

Con el fin de incrementar la solubilidad y la estabilidad de las drogas antineoplásicas, se han propuesto nuevas alternativas de solución, entre ellas, la combinación de los complejos de paladio(II) con compuestos $\beta$-ciclodextrina(29), para mejorar su actividad biológica con respecto a otras drogas de uso clínico (30).Tal es así, que en un trabajo previo, se prepararon los complejos de paladio(II), Pd(MePhPzTSC) y $\mathrm{Pd}\left(\mathrm{Ph}_{2} \mathrm{PzTSC}\right)_{2}$, los cuales fueron incluidos en la $\beta$-ciclodextrina y ensayados en la línea celular de adenocarcinoma de mama (MCF7), y se evidenció que cuando se incluyen los complejos de paladio(II) con ligandos tiosemicarbazonas en la $\beta$-ciclodextrina, aumenta significativamente la actividad antiproliferativa frente a la línea celular MCF-7 (20), es por ello que en este estudio se procedió a evaluar la actividad antiproliferativa in vitro de los complejos de paladio(II) con ligandos tiosemicarbazonas incluidos en $\beta$-ciclodextrina, frente a tres diferentes líneas celulares humanas, pertenecientes a diferentes tumores, tales como la de adenocarcinoma de próstata (PC-3), la de cérvix uterino (HeLa) y la de colon (HCT-15).

\section{MATERIALES Y MÉTODOS}

\section{Ligandos y complejos de paladio(II)}

Se utilizaron los siguientes ligandos: 3-metil-1-fenil-1-pirazol-4-carboxaldehido tiosemicarbazona (MePhPzTSC) o $\left(\mathrm{HL}^{1}\right)$ y 1,3-difenilpirazol-4-carboxaldehido tiosemicarbazona( $\left.\mathrm{Ph}_{2} \mathrm{PzTSC}\right)$ o $\left(\mathrm{HL}^{2}\right)$; así como los complejos de paladio(II)Pd(MePhPzTSC) $)_{2} \mathrm{O}$ $\operatorname{Pd}\left(\mathrm{L}^{1}\right)_{2}$ y $\operatorname{Pd}\left(\mathrm{Ph}_{2} \mathrm{PzTSC}\right)_{2}$ o $\mathrm{Pd}\left(\mathrm{L}^{2}\right)_{2}$. Además, se utilizaron los complejos $\mathrm{Pd} /$ ligandos incluidos en la $\beta$-ciclodextrina $(\beta-\mathrm{CD}), \operatorname{Pd}(\mathrm{MePhPzTSC})_{2}$ ] - $3-\mathrm{CD}$ y $\mathrm{Pd}\left(\mathrm{Ph}_{2} \mathrm{PzTSC}\right)_{2} \bullet \beta-\mathrm{CD}$ (Fig. 1 a y b). Todos estos compuestos fueron sintetizados $y$ caracterizados por el grupo de Investigación en Química de Coordinación y Bioinorgánica de la Universidad Nacional de Colombia. Para la preparación del compuesto $\mathrm{Pd}(\mathrm{MePhPzTSC}) \cdot \beta-$ $\mathrm{CD}$, se mezclaron $15 \mathrm{mg}$ de $\mathrm{Pd}(\mathrm{MePhPzTSC})_{2} \mathrm{y}$ 27,35 $\mathrm{mg}$ de $\beta-C D$ (relación molar de 1:1) en agua, con agitación constante y a temperatura ambiente por 24 horas, mientras que para la preparación del compuesto $\mathrm{Pd}\left(\mathrm{Ph}_{2} \mathrm{PzTSC}_{2} \bullet \beta-\right.$ $\mathrm{CD}$, se mezclaron $15 \mathrm{mg}$ de $\left[\mathrm{Pd}\left(\mathrm{Ph}_{2} \mathrm{PzTSC}_{2}\right]\right.$ y $22,8 \mathrm{mg}$ de $\beta-C D$ (relación molar de 1:1) en un mortero de ágata hasta obtener un polvo homogéneo $(31,32)$.

\section{Líneas de células tumorales empleadas y cultivo celular}

Se emplearon tres líneas celulares: Las líneas celulares HCT-15(línea celular humana de adenocarcinoma de colon), PC-3 (línea celular humana de adenocarcinoma de próstata) y HeLa (línea celular humana de adenocarcinoma de cérvix uterino). Estos diferentes tipos de células tumorales fueron cultivados en medio RPMI1640 con una solución $0,05 \%$ de antibiótico y suplementado con suero fetal bovino al 10\%, hasta obtener una confluencia del $80 \%$. Las células se disociaron con una solución de tripsina al $0,25 \%$ y luego se sembraron por triplicado en placas de cultivo de 96 pozos, a una densidad de 20,000 células/100 $\mu \mathrm{L}$ por pozo. Luego de permitir la adhesión celular durante 24 horas, 

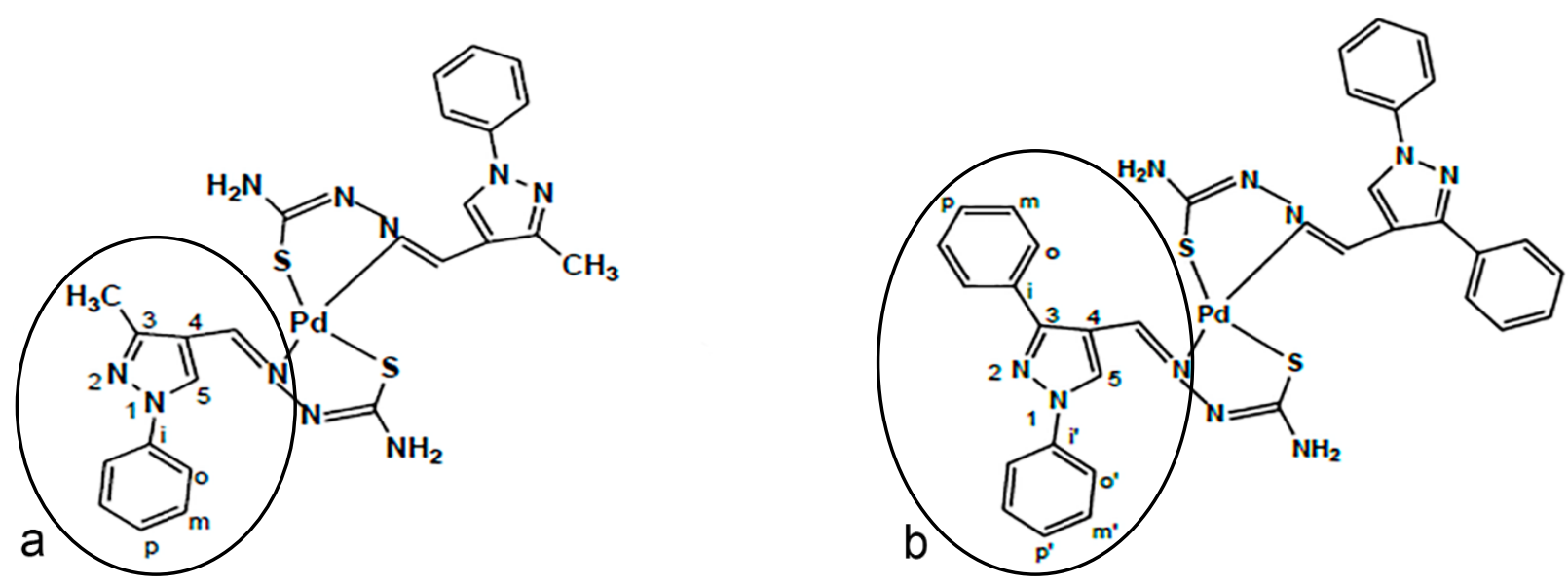

Fig. 1. Los complejos de paladio(II) a. $\left[\mathrm{Pd}(\mathrm{MePhPzTSC})_{2}\right]$ o $\left[\mathrm{Pd}\left(\mathrm{L}^{1}\right)_{2}\right]$ y b. $\left[\mathrm{Pd}\left(\mathrm{Ph}_{2} \mathrm{PzTSC}\right)_{2}\right]$ o $\left[\mathrm{Pd}\left(\mathrm{L}^{2}\right)_{2}\right]$. a. El óvalo resalta el ligando 3-metil-1-fenil-1-pirazol-4carboxaldehido tiosemicarbazona (MePhPzTSC) o $\left(\mathrm{HL}^{1}\right)$ y b. El óvalo resalta el ligando 1,3-difenilpirazol-4-carboxaldehido tiosemicarbazona $\left(\mathrm{Ph}_{2} \mathrm{PzTSC}\right) \mathrm{o}$ $\left(\mathrm{HL}^{2}\right)$.

se adicionaron los compuestos en un rango de concentraciones de 0,1 a $10 \mu \mathrm{M}$.

\section{Evaluación de la actividad antiproliferativa mediante el ensayo de la sulforodamina $B$}

Después de 24 horas de exposición con los compuestos, se evaluó la viabilidad celular por el método de la sulforodamina B (SRB) (33). Las células se fijaron con $100 \mu \mathrm{L}$ de $\mathrm{CCl}_{3} \mathrm{COOH}$ al $10 \%$ y se tiñeron durante 30 minutos con $50 \mu \mathrm{L}$ de la solución de SRB al $0,4 \%$ en ácido acético al $1 \%$. Se procedió a lavar las placas 5 veces con una solución de $\mathrm{CH}_{3} \mathrm{COOH}$ al $1 \%$ y posteriormente se extrajo el colorante celular, utilizando $100 \mu \mathrm{L}$ de una solución Tris Base $10 \mathrm{mM}(\mathrm{pH} 10,5)$ durante 5 minutos con agitación suave. Finalmente, se determinó la densidad óptica de la solución a $556 \mathrm{~nm}$, mediante un espectrofotómetro Organon Teknica Microwell System, Durham, NC, USA. El efecto antiproliferativo de los compuestos sobre las células HCT-15, PC-3 y HeLa, se expresó como valores del porcentaje de inhibición, usando la siguiente fórmula: \% Inhibición $=[1-($ absorbancia media de células tratadas/absorbancia media del control)] x 100.

\section{Cálculo de la concentración inhibitoria}

La concentración inhibitoria, se define como la concentración micromolar requerida de los compuestos ensayados para inhibir al $50 \%$ el crecimiento de las células tumorales. La concentración inhibitoria $\left(\mathrm{CI}_{50}\right)$ se determinó a partir de las curvas de inhibición del porcentaje 
vs la concentración. Se seleccionó el modelo no lineal hiperbólico para el ajuste de los datos. El análisis de regresión no lineal que se implementó, permitió la obtención de los parámetros del modelo mediante el método de los mínimos cuadrados, usando como variable respuesta a la dosis inhibitoria y como variable de regresión a la concentración de los compuestos evaluados. La selección del modelo se basó en la gran utilidad del mismo para este tipo de ensayos y por tener el más bajo AIC (Criterio de información de Akaike). Adicionalmente, se aplicaron los estadísticos convencionales con el programa Prism 5, versión 5.03, a fin de obtener los valores de la desviación estándar y la aplicación de la t de Student.

\section{RESULTADOS}

Los valores $\mathrm{CI}_{50}$ obtenidos para los compuestos estudiados se incluyen en la Tabla I. En las Figs. 2a y 2 b se muestra que los valores de $\mathrm{CI}_{50}$ disminuyeron significativamente cuando los ligandos se encontraban coordinados con el ion $\mathrm{Pd}(\mathrm{II})$, tal como se observó para los complejos $\left[\mathrm{Pd}(\mathrm{MePhPzTSC})_{2}\right]\left(\mathrm{CI}_{50}=0,45\right.$ $1,13 \mu \mathrm{M})(\mathrm{p}<0,04)$ y $\left[\mathrm{Pd}\left(\mathrm{Ph}_{2} \mathrm{PzTSC}\right)_{2}\right]\left(\mathrm{CI}_{50}=\right.$ $0,86-1,31 \mu \mathrm{M})(\mathrm{p}<0,05)$, mientras que cuando estos complejos se encontraban incluidos en la $\beta-C D$, los valores de $\mathrm{CI}_{50}$ se redujeron en el rango de $0,14-0,52 \mu \mathrm{M}$ para los compuestos $\left[\mathrm{Pd}(\mathrm{MePhPzTSC})_{2}\right] \quad \bullet \beta-\mathrm{CD} \quad(\mathrm{p}<0,001) \quad$ (Fig. 2a) y $\left[\mathrm{Pd}\left(\mathrm{Ph}_{2} \mathrm{PzTSC}\right)_{2}\right] \cdot \beta-\mathrm{CD}(\mathrm{p}<0,001)$ (Fig. $2 b)$. A pesar de que en algunas condiciones se evidenciaron variaciones en la respuesta a algunos compuestos, esto no afectó la inhibición pronunciada de la respuesta proliferativa observada especialmente cuando se incorporó la $ß-C D$. Estos resultados indican que se requiere aproximadamente de 5 a 10 veces menos la concentración de los complejos de paladio(II) solos que cuando estos complejos se incluyen en la ß-CD (Tabla I).En el caso del compuesto $\left[\mathrm{Pd}(\mathrm{MePhPzTSC})_{2}\right] \cdot ß-\mathrm{CD}$, el valor de $\mathrm{CI}_{50}$ obtenido fue menor con respecto al complejo sin incluir: PC-3 (7 veces menor), HeLa (7 veces menor) y HCT-15 (6 veces menor). En el caso del compuesto $\left[\mathrm{Pd}\left(\mathrm{Ph}_{2} \mathrm{PzTSC}_{2}\right] \cdot \beta-\mathrm{CD}\right.$, el valor de $\mathrm{CI}_{50}$ fue 6 veces menor para todas las líneas evaluadas. Finalmente, la inclusión de los complejos de paladio(II) con ligandos tiosemicarbazonas en la $\beta-C D$, incrementó su actividad antiproliferante.

Los valores de $\mathrm{CI}_{50}$ obtenidos para el compuesto $\left[\mathrm{Pd}(\mathrm{MePhPzTSC})_{2}\right] \bullet \beta-\mathrm{CD}$ mostraron una tendencia ascendente de $0,16 \mu \mathrm{M}$ para la línea HeLa, mientras que para el compuesto $\left[\mathrm{Pd}\left(\mathrm{Ph}_{2} \mathrm{PzTSC}\right)_{2}\right] \cdot \beta-\mathrm{CD}$, se evidenció un valor de $\mathrm{CI}_{50}$ ascendente a $0,34 \mu \mathrm{M}$ para la línea HCT-15. Finalmente, a pesar de que la línea celular HeLa fue más susceptibles que otras con respecto a los complejos de paladio(II) incluidos en $\beta-C D$, en todos los casos la inclusión de estos complejos en la ciclodextrina, originó una disminución de los valores $\mathrm{CI}_{50}$ de 5 a 10 veces (Tabla I). La línea celular HeLa mostró mayor susceptibilidad al compuesto $\left[\mathrm{Pd}(\mathrm{MePhPzTSC})_{2}\right] \cdot \beta-\mathrm{CD}$, mientras que la línea HCT-15 mostró mayor susceptibilidad al compuesto $\left[\mathrm{Pd}\left(\mathrm{Ph}_{2} \mathrm{PzTSC}\right)_{2}\right] \bullet ß-\mathrm{CD}$.

\section{DISCUSIÓN}

En este estudio, se investigó el efecto antiproliferativo de los ligandos $\left(\mathrm{HL}^{1-2}\right)$, los complejos bis-quelatos de paladio(II) $\left[\mathrm{Pd}\left(\mathrm{L}^{1}\right)_{2}\right]$ y los complejos $\left[\mathrm{Pd}\left(\mathrm{L}^{2}\right)_{2}\right]$ incluidos en $B-\mathrm{CD}$. Estudios previos han indicado que la línea celular de cáncer de mama (MCF-7), es susceptible a estos compuestos, así como lo es al cisplatino 
TABLA I.

VALORES DE $\mathrm{CI}_{50}(\mu \mathrm{M})$ DE LOS LIGANDOS TIOSEMICARBAZONAS, LOS COMPLEJOS DE PALADIO(II) ([PD(MePhPzTSC $\left.)_{2}\right] \quad Y \quad\left[\mathrm{PD}\left(\mathrm{Ph}_{2} \mathrm{PzTSC}_{2}\right] \quad \mathrm{Y}\right.$ LOS COMPLEJOS DE PALADIO(II) INCLUIDOS EN LA B-CICLODEXTRINA FRENTE A LAS LÍNEAS DE CÉLULAS TUMORALES DE HUMANO, HCT-15, PC-3 Y HeLa.

\begin{tabular}{llll}
\hline Compuestos & HCT-15 & PC-3 & HeLa \\
\hline$(\mathrm{MePhPzTSC}) \mathrm{o} \mathrm{HL}^{1}$ & $2,7 \pm 1,7$ & $2,0 \pm 2$ & $1,1 \pm 0,7$ \\
{$\left[\mathrm{Pd}(\mathrm{MePhPzTSC})_{2}\right]$ o $\left[\mathrm{Pd}\left(\mathrm{L}^{1}\right)_{2}\right]$} & $1,13 \pm 2,2$ & $0,82 \pm 1,7$ & $0,45 \pm 0,8$ \\
{$\left[\mathrm{Pd}(\mathrm{MePhPzTSC})_{2}\right] \cdot ß-\mathrm{CD}$} & $0,40 \pm 1,4$ & $0,29 \pm 2,7$ & $0,16 \pm 0,5$ \\
$\left(\mathrm{Ph}{ }_{2} \mathrm{PzTSC}\right)$ o HL & $2,0 \pm 0,31$ & $3,0 \pm 0,7$ & $3,1 \pm 0,9$ \\
{$\left[\mathrm{Pd}\left(\mathrm{Ph}_{2} \mathrm{PzTSC}_{2}\right]\right.$ o $\left[\mathrm{Pd}\left(\mathrm{L}^{2}\right)_{2}\right]$} & $0,86 \pm 1,1$ & $1,23 \pm 1,4$ & $1,31 \pm 1,8$ \\
{$\left[\mathrm{Pd}\left(\mathrm{Ph}_{2} \mathrm{PzTSC}\right)_{2}\right] \cdot ß-\mathrm{CD}$} & $0,34 \pm 1,1$ & $0,49 \pm 0,5$ & $0,52 \pm 1,5$ \\
\hline
\end{tabular}

*Los valores están expresados como promedio $\pm \mathrm{DE}$

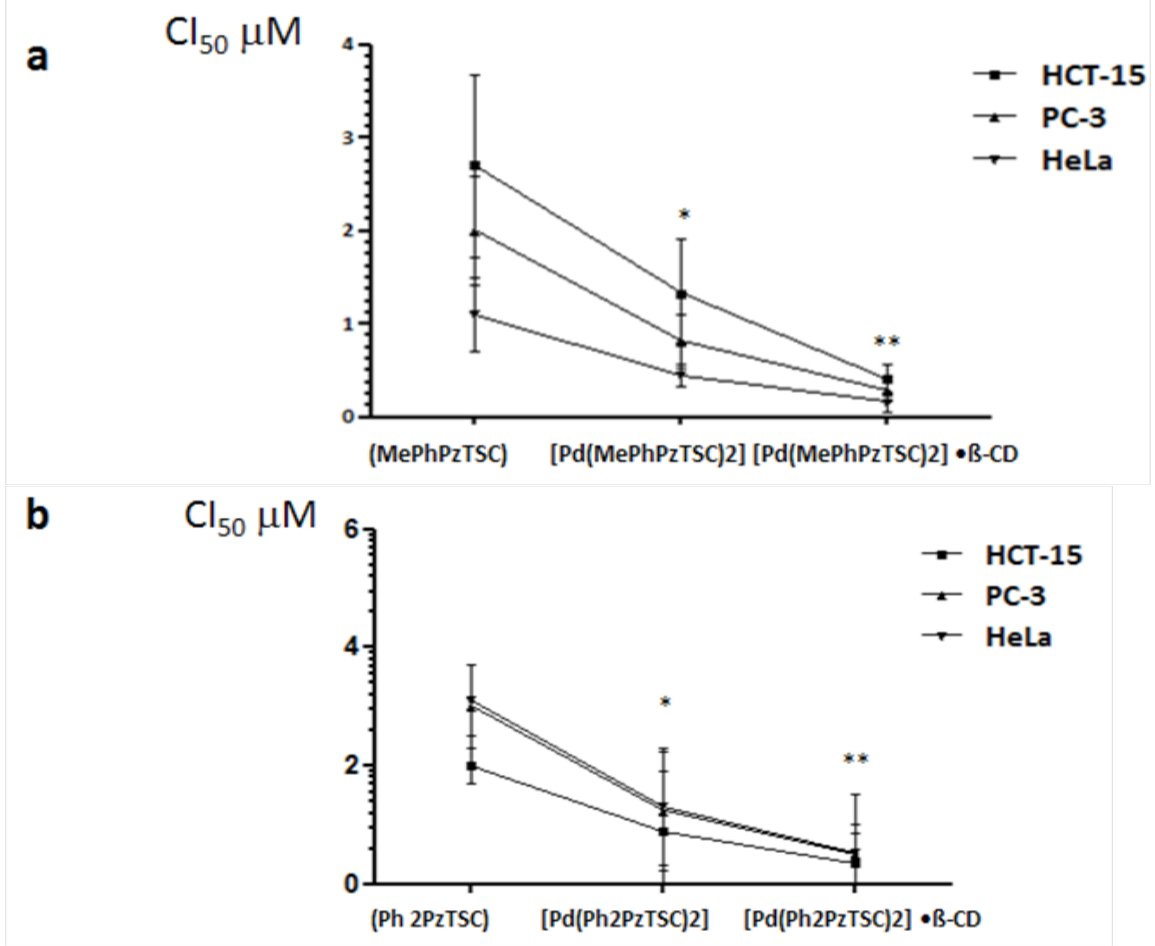

Fig.2. a) Efecto antiproliferativo del ligando MePhPzTSC, su respectivo complejo de paladio(II) y este complejo incluido en la $\beta$-ciclodextrina, frente a diferentes líneas de células tumorales. b) Efecto antiproliferativodel ligando $\mathrm{Ph}_{2} \mathrm{PzTSC}$, su respectivo complejo de paladio(II) y este complejo incluido en la $\beta$ ciclodextrina, frente a diferentes líneas de células tumorales. ${ }^{*} \mathrm{p}<0,05 \mathrm{y}{ }^{* *} \mathrm{p}<0,001$. Cada condicion fue evaluada por triplicado

Vol. 59(1): 17 - 27, 2018 
(20), debido a ello, en este estudio se utilizaron tres diferentes líneas de células tumorales celulares para evaluar el efecto antiproliferativo de los compuestos en mención .A pesar de que en algunos casos los valores de las $\mathrm{DE}$ fueron mayores a la media, producto posiblemente a la "n" limitada, los resultados evidenciaron que con la inclusión de los complejos [Pd(MePhPzTSC $\left.)_{2}\right]$ y $\left[\mathrm{Pd}\left(\mathrm{Ph}_{2} \mathrm{PzTSC}\right)_{2}\right]$ en la $\beta-C D$, se obtienen menores concentraciones micromolares de inhibición $\left(\mathrm{CI}_{50}\right)$, incrementando así su actividad antiproliferativa, efecto que pudo observarse en las 3 líneas de células tumorales evaluadas en este trabajo. Los resultados obtenidos respecto a los valores de $\mathrm{CI}_{50}$, se mantuvieron bajos, en comparación con los altos rangos de valores $\mathrm{CI}_{50}$ informados para los complejos de paladio(II) evaluados en las líneas $\mathrm{MCF}-7\left(\mathrm{CI}_{50}=25,09\right.$ $48,0 \mu \mathrm{M}), \mathrm{PC}-3\left(\mathrm{CI}_{50}=0,08-72,2 \mu \mathrm{M}\right)$ y HeLa $\left(\mathrm{CI}_{50}=1,0-22,2 \mu \mathrm{M}\right)(1,2,7,9,14,18)$.

Tanto las tiosemicarbazonas como los complejos de paladio(II) fueron altamente tóxicos para las líneas celulares PC-3, HeLa y HCT-15, efecto que puede estar relacionado con la inhibición de la biosíntesis del ADN, mediante el bloqueo de la enzima ribonucleótido reductasa, esencial en la conversión de ribonucleótidos en desoxirribonucleótidos, inhibición de la tiorredoxina reductasa involucrada en la en la biosíntesis de nucleótidos o como un agente que se intercala entre las bases pirimidinas y purinas del $\mathrm{ADN}$, induciendo cambios en la conformación de la doble hélice, lo que conduce a la muerte celular $(5,7,9,10)$, o al bloqueo de la división de las células cancerosas (8). Otros modos de acción descritos recientemente para los complejos de paladio(II), pueden estar relacionados con la inhibición de la entrada al ciclo celular e inducción de la apoptosis de las células tumorales (24).

Por otro lado, los resultados obtenidos sugieren que los complejos de paladio(II) con sus ligandos tiosemicarbazonas y estos complejos de paladio(II) incluidos en la $\beta$-ciclodextrina, pueden ser considerados como posibles agentes quimioterápeúticos, debido a su potencial efecto antiproliferante sobre células neoplásicas representadas en las líneas celulares PC-3, HeLa y HCT-15. Además, estos compuestos ofrecen la posibilidad de reducir los efectos tóxicos observados con las diferentes quimioterapias disponibles en la actualidad, por cuanto para alcanzar valores aceptables de la $\mathrm{CI}_{50}$, se logró reducir la concentración óptima de los complejos de paladio(II) incluidos en la $\beta$-CD, en comparación con los ligandos tiosemicarbazonas libres y sus respectivos complejos de paladio(II).

En general, en este estudio se evidenció que la actividad antiproliferativa in vitro de los complejos de paladio(II) incluidos en la $\beta$-ciclodextrina frente a las líneas tumorales ensayadas, se incrementa debido a los bajos valores de $\mathrm{CI}_{50}$ obtenidos. La línea celular HeLa muestran mayor susceptibilidad al compuesto $\mathrm{Pd}(\mathrm{MePhPzTSC})_{2} \cdot \beta-\mathrm{CD}$, mientras que la línea HCT-15 al compuestoPd( $\left.\mathrm{Ph}_{2} \mathrm{PzTSC}\right)_{2} \bullet ß-$ CD (Tabla I). Basados en los informes de investigaciones previas y de otros investigadores, la implementación de la $\beta$-ciclodextrina como medio de transporte puede ser un factor muy importante en el campo de la oncología (25-30). Se deberían realizar estudios complementarios respecto al modo de acción de los complejos de paladio(II) y los complejos incluidos en la $\beta$-ciclodextrina, con la finalidad de conocer que factores son los que inducen el incremento de la actividad antiproliferante frente a las líneas 
tumorales ensayadas y otras por investigar. Además, los resultados obtenidos servirán para proponer su uso como un potencial agente quimioterapéutico en los diversos tipos de tumores.

\section{AGRADECIMIENTOS}

Los autores agradecen a todos los miembros del grupo de investigación en Química de Coordinación y Bioinorgánica, Universidad Nacional de Colombia por la síntesis y caracterización de los complejos de paladio(II), al Centro de Microscopía Electrónica "Dr. Ernesto Palacios Prü", del Instituto de Inmunología Clínica, Facultad de Medicina, Universidad de Los Andes- Mérida, Venezuela y al Centro Integral de Hematología y Oncología Médica C.A. por su apoyo y colaboración en el desarrollo de la investigación.

\section{REFERENCIAS}

1. Queiroz MJ, Peixoto D, Calhelha RC, Soares P, Dos Santos T, Lima RT, Campos JF, Abreu RM, Ferreira IC, Vasconcelos MH. New di(hetero)arylethers and di(hetero)arylamines in the thieno[3,2-b] pyridine series: synthesis, growth inhibitory activity on human tumor cell lines and nontumor cells, effects on cell cycle and on programmed cell death. Eur J Med Chem 2013; 69:855-862.

2. Matesanz AI, Perles J, Souza P. New palladium and platinum complexes with bioactive 3,5-diacetyl-1,2,4-triazol bis(4cyclohexyl thiosemicarbazone) ligand: chemistry, antiproliferative activity and preliminary toxicity studies. Dalton Trans 2012;41(40):12538-12547.

3. Graham RD WD. The synthesis and screening for anti-bacterial, -cancer, -fungicidal and -viral activities of some complexes of palladium and nickel. J Inorg Nucl Chem 1979;41:1245-1249.

4. Abu-SurrahAS, Abdalla MY. Palladiumbased chemotherapeutic agents: Routes toward complexes with good antitumor activity. Cancer Ther 2008;6:1-10.

5. Finch RA, Liu M, Grill SP, Rose WC, Loomis R, Vasquez KM,Cheng Y, Sartorelli AC. Triapine (3-aminopyridine2-carboxaldehyde- thiosemicarbazone): A potent inhibitor of ribonucleotide reductase activity with broad spectrum antitumor activity. Biochem Pharmacol 2000;15;59(8):983-991.

6. Sengupta SK, Pandey OP, Srivastava BK. Synthesis, structural and biochemical aspects of titanocene and zirconocene chelates of acetylferrocenyl thiosemicarbazones.Trans Met Chem 1998;23:349-353.

7. Ari F, Cevatemre B, Armutak EI, Aztopal N, Yilmaz VT, Ulukaya E. Apoptosis-inducing effect of a palladium(II) saccharinate complex of terpyridine on human breast cancer cells in vitro and in vivo. Bioorg Med Chem 2014;22(17):49484954.

8. Kalaivani P, Prabhakaran R, Dallemer F, Poornima P, Vaishnavi E, Ramachandran E,Padma VV, Renganathan $R$, Natarajan K. DNA, protein binding, cytotoxicity, cellular uptake and antibacterial activities of new palladium(II) complexes of thiosemicarbazone ligands: effects of substitution on biological activity. Metallomics 2012;4(1):101-113.

9. Matesanz AI, Leitao I, Souza P. Palladium(II) and platinum(II) bis(thiosemicarbazone) complexes of the 2,6-diacetylpyridine series with high cytotoxic activity in cisplatin resistant A2780cisR tumor cells and reduced toxicity.

Vol. 59(1): 17 - 27, 2018 
J Inorg Biochem 2013;125:26-31.

10. Shao J, Zhou B, Chu B, Yen Y. Ribonucleotide reductase inhibitors and future drug design. Curr Cancer Drug Targets 2006;6(5):409-431.

11. Dodoff NI KM, Kuduk-Jaworska J, Mastalarz A, Kochel A, Vladimira Vassilieva V, Vassilev $N$, Trendafilova N, Georgieva I, Lalia-Kantouri M, Apostolova M. Structure, NMR spectra and cytotoxic effect of palladium(II) and platinum(II) complexes of glyoxylic Acid oxime. Chemija 2009;20: 208-217.

12. Jagadeesh M, Lavanya M, Kalangi SK, Sarala Y, Ramachandraiah C, Varada Reddy A. Spectroscopic characterization, antioxidant and antitumour studies of novel bromo substituted thiosemicarbazone and its copper(II), nickel(II) and palladium(II) complexes. Spectrochim Acta A Mol Biomol Spectrosc 2015;25(135):180-184.

13. Prabhakaran R, Kalaivani $P$, Poornima P, Dallemer F, Huang R, Vijaya Padma V,Natarajan K. Synthesis, DNA/protein binding and in vitro cytotoxic studies of new palladium metallothiosemicarbazones. Bioorg Med Chem 2013;21(21):6742-6752.

14. Ari F, Aztopal N, Icsel C, Yilmaz VT, Guney E, Buyukgungor O, Ulukaya E. Synthesis, structural characterization and cell death-inducing effect of novel palladium(II) and platinum(II) saccharinate complexes with 2-(hydroxymethyl) pyridine and 2-(2-hydroxyethyl)pyridine on cancer cells in vitro. Bioorg Med Chem 2013;21(21):6427-6434.

15. Hernández W, Paz J, Vaisberg A, Spodine E, Richter R, Beyer L. Synthesis, characterization, and in vitro cytotoxic activities of benzaldehyde thiosemicarbazone derivatives and their palladium (II) and platinum (II) complexes against various human tumor cell lines.Bioinorg Chem Appl
2008; 2008:1-9.

16. Ramachandran E, Kalaivani P, Prabhakaran R, Rath NP, Brinda S, Poornima P, PadmaV, Natarajan $K$. Synthesis, X-ray crystal structure, DNA binding, antioxidant and cytotoxicity studies of $\mathrm{Ni}$ (II) and $\mathrm{Pd}(\mathrm{II})$ thiosemicarbazone complexes. Metallomics 2012;4(2):218227.

17. Rosu T, Pahontu E, Pasculescu S, Georgescu R, Stanica N, Curaj A, Popescu A, Leabu M. Synthesis, characterization antibacterial and antiproliferative activity of novel $\mathrm{Cu}$ (II) and $\mathrm{Pd}(\mathrm{II})$ complexes with 2-hydroxy-8-R-tricyclo[7.3.1.0.2,7] tridecane-13-one thiosemicarbazone. Eur J Med Chem 2010;45(4):1627-1634.

18. Ulukaya E, Ari F, Dimas K, Ikitimur EI, Guney E, Yilmaz VT. Anti-cancer activity of a novel palladium(II) complex on human breast cancer cells in vitro and in vivo.Eur $\mathrm{J}$ Med Chem 2011;46(10):4957-4963.

19. Kacar O, Adiguzel Z, Yilmaz VT, Cetin Y, Cevatemre B, Arda N, Baykal A T, Ulukaya E, Acilan C. Evaluation of the molecular mechanisms of a palladium(II) saccharinate complex with terpyridine as an anticancer agent. Anticancer Drugs 2014;25(1):17-29.

20. Jaimes N, Salmen S, Colmenares MC, Burgos AE, Tamayo L, Mendoza RV, Cantor A. Cytotoxic effect of palladium(II) inclusion compounds in beta-cyclodextrin. Biomedica 2016;36(4):603-611.

21. Lingen V, Lüning $A$, Krest $A$, Deacon GB, Schur J, Ott I, Pantenburg I, Meyer G, Klein A. Labile Pd-sulphur and Ptsulphur bonds in organometallic palladium and platinum complexes [(COD)M(alkyl) (S-ligand)]n+-A speciation study. J Inorg Biochem 2016;165:119-127.

22. Roche M, Bignon J, Brion J D, Hamze A, Alami M. Tandem one-pot palladium- 
catalyzed coupling of hydrazones, haloindoles, and amines: synthesis of amino-N-vinylindoles and their effect on human colon carcinoma cells. J Org Chem 2014;79(16):7583-7592.

23. Khan H, Badshah A, Murtaz G, Said M, Rehman ZU, Neuhausen C, Todorova M, Jean-Claude BJ, Butler IS. Synthesis, characterization and anticancer studies of mixed ligand dithiocarbamate palladium(II) complexes. Eur J Med Chem 2011;46(9):4071-4077.

24. Kacar O, Cevatemre B, Hatipoglu I, Arda N, Ulukaya E, Yilmaz VT, Acilan C. The role of cell cycle progression for the apoptosis of cancer cells induced by palladium(II)-saccharinate complexes of terpyridine. Bioorg Med Chem 2017; 25 (6):1770-1777.

25. Gidwani B, Vyas A. A comprehensive review on cyclodextrin-based carriers for delivery of chemotherapeutic cytotoxic anticancer drugs. Biomed Res Int 2015;2015:1-15.

26. Jiang RJ, Zhao YL, Chen YJ, Xiao D, Wang F, Han B, Yang J, Liao XL, Yang LJ, Gao CZ, Yang B. Synthesis, characterization, and in vitro evaluation of artesunate-beta-cyclodextrin conjugates as novel anti-cancer prodrugs. Carbohydr Res 2014;400:19-25.

27. Sudakaran SV, Venugopal JR, Vijayakumar GP, Abisegapriyan S, Grace AN, Ramakrishna S. Sequel of $\mathrm{MgO}$ nanoparticles in PLACL nanofibers for anticancer therapy in synergy with curcumin/ beta-cyclodextrin. Mater Sci Eng C Mater Biol Appl2017;71:620-628.

28. Yoon DY, Kim JC. In vivo lifetime and anti-cancer efficacy of doxorubicin-loaded nanogels composed of cinnamoyl poly (betacyclodextrin) and cinnamoyl Pluronic F127. J Biomater Sci Polym Ed2017;28(6):505518.
29. Xu Z, Zhang Y, Hu Q, Tang Q, Xu J, Wu J, Brett K, Ma D, Xue W. Biocompatible hyperbranched polyglycerol modified betacyclodextrin derivatives for docetaxel delivery. Mater Sci Eng C Mater Biol Appl2017;71:965-972.

30. LoftssonT, DuchêneD. Cyclodextrins and their pharmaceutical applications.Int $\mathbf{J}$ Pharm.2007; 329:1-11.

31. Burgos C AE, Tamayo L, TorrellasHidalgo R. Synthesis, characterization and antimicrobial activity of a $\mathrm{Pd}(\mathrm{II})$ complex with a 1,3-diphenylpyrazole-4carboxaldehyde thiosemicarbazone ligand. Rev Udcaactual Divulg Cient 2014;17:477486.

32. Tamayo LV, Burgos AE, Brandão PFB. Synthesis, characterization, andantimicrobial activity of the ligand 3-methylpyrazole-4carboxaldehyde thiosemicarbazone and its Pd(II) complex. Phosphorus Sulfur Silicon Relat Elem 2013;189:52-59.

33. Skehan P, Storeng R, Scudiero D, Monks A, McMahon J, Vistica D, Warren J T, Bokesch H, Kenney S, Boyd M R. New colorimetric cytotoxicity assay for anticancer-drug screening. J Natl Cancer Inst 1990;82(13):1107-1112. 